\title{
An Empirical Investigation of User Term Feedback in Text-based Targeted Image Search
}

\author{
JOYCE Y. CHAI \\ CHEN ZHANG \\ RONG JIN
}

\author{
Department of Computer Science and Engineering \\ Michigan State University
}

\begin{abstract}
Text queries are natural and intuitive for users to describe their information needs. However, text-based image retrieval faces many challenges. Traditional text retrieval techniques on image descriptions have not been very successful. This is mainly due to the inconsistent textual descriptions and the discrepancies between user queries and terms in the descriptions. To investigate strategies to alleviate this vocabulary problem, this paper examines the role of user term feedback in targeted image search that is based on text-based image retrieval. Term feedback refers to the feedback from a user on specific terms regarding their relevance to a target image. Previous studies have indicated the effectiveness of term feedback in interactive text retrieval. However, in our experiments on text-based image retrieval, the term feedback has not shown to be effective. Our results indicate that, although term feedback has a positive effect by allowing users to identify more relevant terms, it also has a strong negative effect by providing more opportunities for users to specify irrelevant terms. To understand these different effects and their implications, this paper further analyzes important factors that contribute to the utility of term feedback and discusses the outlook of term feedback in interactive text-based image retrieval.
\end{abstract}

Categories and Subject Descriptors: H.3.3 [Information Search and Retrieval]: Query Formulation, Relevance Feedback, Search Process; H.5.2 [User Interfaces]: Interaction Styles

General Terms: Experimentation, performance, Human Factors

Additional Key Words and Phrases: Text-based interactive image retrieval, user term feedback

\section{INTRODUCTION}

Given the tremendous amount of image data available to all walks of life, it has become increasingly important to equip computer systems with capabilities to efficiently and effectively retrieve images of user interests. To address this need, previous work on image retrieval has mainly focused on two general approaches: content-based approaches [Faloutsos et al. 1994; Enser 1995; Feder 1996; Pent-

Contact address: Joyce Y. Chai, 3115 Engineering Building, Department of Computer Science and Engineering, Michigan State University, East Lansing, MI 48824.

Permission to make digital/hard copy of all or part of this material without fee for personal or classroom use provided that the copies are not made or distributed for profit or commercial advantage, the ACM copyright/server notice, the title of the publication, and its date appear, and notice is given that copying is by permission of the ACM, Inc. To copy otherwise, to republish, to post on servers, or to redistribute to lists requires prior specific permission and/or a fee.

(C) 2006 ACM 1529-3785/2006/0700-0001 $\$ 5.00$ 
land et al. 1996; Forsyth 1997; Ravela and Manmatha 1998; Evgeniou et al. 2003; Chang et al. 2003; Chen et al. 2005; He et al. 2004; Hoi and Lyu 2004a; Jeon et al. 2003a] and text-based approaches [Tamura and Yokoya 1984; Chang and Hsu 1992; Wactlar et al. 1996; Frankel et al. 1997; Rui et al. 1999; Bjarnestam 1998]. The text-based approaches apply traditional text retrieval techniques to image annotations or descriptions. The content-based approaches apply image processing techniques to first extract image features and then retrieve relevant images based on the match of these features.

Despite tremendous improvement in content-based retrieval, the content-based approaches still have many limitations. First, it is difficult for users to specify visual queries with low-level visual features. Often, specific tools are required to support queries with rudimentary sketches or paintings [McDonald and Tait 2003]. Second, low level image features cannot precisely describe user information needs. There is a gap between low-level visual descriptions and a user's semantic expectation [Santini 2001]. Text queries, on the other hand, are more intuitive and natural for users to specify their information needs.

However, text-based image retrieval also faces many challenges. One major problem is that the task of describing image content is highly subjective. The perspective of textual descriptions given by an annotator could be different from the perspective of a user. A picture can mean different things to different people. It can also mean different things to the same person at different time. Furthermore, even with the same view, the words used to describe the content could vary from one person to another [Keister 1994]. In other words, there could be a variety of inconsistencies between user textual queries and image annotations or descriptions.

To alleviate the inconsistency problem, different strategies can be potentially applied, for example, showing textual descriptions of similar images to shape user's query terms; and asking users for relevance feedback concerning the retrieved images. In this paper, rather than investigating these different strategies, we focus on the role of term feedback in interactive image retrieval. Term feedback refers to the feedback from a user on specific terms regarding their relevance to the target information. Previous studies have shown that user term feedback has been effective in interactive text retrieval. In particular, the feedback acquired from users can improve the system final retrieval performance [Koenemann and Belkin 1996]. Therefore our focus in this paper is on whether the use of term feedback can be effective in interactive text-based image retrieval.

Our hypothesis is that users should be more responsive to the terms prompted by the system. It would be easier for users to select terms from alternatives than to find terms to describe their target images. Furthermore, the selected terms could bridge the vocabulary discrepancy between image annotations and query terms. Thus the selected terms could potentially improve the quality of query expansion, which would ultimately improve the final retrieval results. Based on these hypotheses, we conducted a series of user studies and simulation studies.

-We first conducted a user study (Section 3) to compare the effectiveness of two user interfaces: one interface that requires a user to manually refine search queries (e.g., manual refinement interface) and another interface that solicits feedback from the user about the system generated query terms (e.g., term feedback in- 
terface). This study was conducted based on the guidelines provided by ImageCLEF2004 Interactive Track ${ }^{1}$. This study generated some surprising results that are opposite from our original hypothesis.

- To further understand the role of term feedback, we conducted a series of simulation studies (Section 4). In particular, we focused on the important factors that affect the utility of term feedback including term selection (Section 4.1) and term generation (Section 4.2). These simulation studies allowed us to gain some understanding of the potential of term feedback in interactive text-based image retrieval.

In the rest of this paper, we will describe these studies, present our empirical findings, analyze important factors that contribute to the utility of term feedback, and discuss the potential of term feedback in interactive text-based image retrieval.

\section{RELATED WORK}

There are two general approaches for image retrieval: content-based approaches and text-based approaches. Recently, there have been several studies that combine textbased approaches and content-based approaches for more effective image retrieval [Ogle and Stonebraker 1995; Wang et al. 2001].

In a content-based approach, to retrieve images, users provide the system with image examples or sketches. The system then converts the queries into some internal representation of feature vectors with low level image features. The similarities between the feature vectors of the query and the candidate images are calculated and the images with the largest similarities are retrieved. A review on technology and issues related with content-based image retrieval can be found in [Rui et al. 1999; Smeulders et al. 2000]. Despite recent progress, content-based image retrieval is still limited by the semantic gap between the low-level image features and highlevel semantic content of images. Furthermore, it tends to be more difficult for a user to express their information needs (at the semantic level) through an example image or sketch.

Text-based approaches require that images in the image collection have textual descriptions or annotations. Given the textual information, image retrieval can then become text retrieval, where traditional approaches for text retrieval can be applied to match a user's textual query with textual descriptions/annotations. To acquire textual descriptions for images, two approaches can be applied. The first approach acquires descriptions/annotations manually by human annotators. In other words, descriptive keywords are provided manually by a human to describe the semantic content of each image. There has been a significant amount of literature that examines how searchers pose queries to image systems [Shatford Layne 1994] and how subjects choose to describe categorized images [Jorgensen 1996]. For example, Shatford Layne indicates that image indexing work should not only provide access to images based on the attributes of each image, but also provide access to useful grouping of images in the collection [Shatford Layne 1994]. Previous work also suggests that perceptually stimulated attributes, such as literal objects, the human form, color, and location, are most typically used to describe images [Jorgensen

${ }^{1}$ http://ir.shef.ac.uk/imageclef/ 
1996]. In studying user queries to access Library of Congress American Memory photo archive [Choi and Rasmussen 2003], it was found that most queries are described in terms of kind of person, thing, event, or condition depending on location or time. These findings suggest the principle categories of search terms for users in American history, which can further imply the strategies for manual annotation and indexing tools. To improve manual annotation, some research has been focusing on developing more efficient user interfaces for manual annotation [Shneiderman and Kang 2000].

The second approach to acquire text descriptions is through automated image annotation. For example, [Shen et al. 2000] used the rich textual context in a web page to annotate the images in the same web page. [Srihari et al. 2000] extracted special name entities such as people, place, etc. from collated text to automatically index images. Recently, we have seen automated image annotation using machinelearning techniques that learn the correlation between image features and textual words from the examples of annotated images [Blei and Jordan 2003; Jeon et al. 2003b; Lavrenko et al. 2003; Li and Wang 2003; Monay and Gatica-Perez 2003].

Both of these approaches have their advantages and disadvantages. The manual annotation is more accurate, but more subjective and time consuming. The automated annotation is efficient, however, with less accuracy. The work reported in this paper is based on the manual annotation. Because of its subjectivity, there tends to be a gap between annotated terms and user queries. Such inconsistency can hinder the success of the text-based retrieval. Therefore, instead of focusing on indexing and retrieval techniques, the objective of this paper is to investigate the role of term feedback for the remedy of inconsistency through an interactive manner [Zhang et al. 2005].

Information retrieval is an interactive process where users play an important role in finding relevant information [Henniger and Belkin 1996]. Previous studies on interactive text retrieval range from query expansion mechanism [Anick and Tipirneni 1999; Harman 1988] to interface design issues [Belkin and Marchetti 1990; Hearst 1994]. For interactive content-based image retrieval, studies range from visual query construction [McDonald and Tait 2003] to relevance feedback on retrieved images [He et al. 2003; Hoi and Lyu 2004b; Rui et al. 1998; Vendrig et al. 2001]. Relevance feedback is an important concept in interactive information retrieval. The idea is that based on the feedback from a user during the retrieval process about the previously retrieved objects, the system can adjust the query to better represent the user's information needs.

Previous studies on relevance feedback during interactive text retrieval indicate that users prefer to have explicit knowledge about which terms have been added to the query, and also more strongly, users prefer to control which terms to add to the query [Belkin et al. 1996]. In [Koenemann and Belkin 1996], a penetrable relevance feedback is introduced. The penetrable relevance feedback provides the ability to manipulate the output of the relevance feedback prior to query expansion. The execution of the query was delayed and the user was first presented with the list of terms suggested by the feedback. Users had the opportunity to add none, some, or all of the suggested terms prior to the continuation of the query expansion. For example, the user might select only terms that appear to be central to the 
search topic. The results in [Koenemann and Belkin 1996] indicate that users who interacted with the penetrable version of relevance feedback did significantly better than users of the baseline system without such feedback. Furthermore, it has also shown that users like the feedback component since the task of generating terms is replaced by the easier task of term selection [Koenemann and Belkin 1996]. Furthermore, the results have shown that users in the penetrable condition needed fewer iterations to achieve results comparable to, or better than the other feedback conditions. This penetrable relevance feedback has motivated our work in this paper. Given its successful story in the interactive text retrieval, our goal here is to investigate whether the similar penetrable relevance feedback will make text-based image retrieval more effective.

\section{EMPIRICAL STUDY OF TERM FEEDBACK}

We conducted a series of studies to investigate the role of term feedback in interactive image retrieval. The task used in these studies is similar to the task proposed by the CLEF interactive retrieval track [Clough et al. 2003]: finding a target image in mind through interactive search. In this task, a set of images are put aside as target images. Users are asked to interact with the system to find each of these target images one at a time. This task is somewhat different from the traditional image retrieval tasks. The traditional tasks require users to specify general information needs. In this task, a user already has a target image in mind. Searching for this specific target image usually requires more effort from the user to describe the content of the target image. Therefore, this task is particularly suitable for studying term feedback.

\subsection{Retrieval Model}

The backend retrieval system applies a statistical language model for text-based retrieval [Lafferty and Zhai 2001; Lavrenko and Croft 2001; Ponte 1998]. This approach assumes that each description of an image is generated from a random source and its relevance to a given textual query is computed as the likelihood for the underlying random source to generate the query.

The key for a statistical language modeling approach is how to reliably estimate the most likely statistical source for a textual document. A variety of approaches have been applied, including a multinomial distribution for statistical language models [Ponte 1998], a machine translation model for information retrieval [Berger and Lafferty 1999], a relevance-based language model [Lavrenko and Croft 2001], a hidden Markov model for information retrieval [Miller et al. 1999], and a risk minimization framework [Lafferty and Zhai 2001]. In our system, we adopt the multinomial distribution for statistical language models, which is calculated through the maximum likelihood estimation. Furthermore, a Jelinek-Mercer smoothing scheme is used to alleviate the sparse data problem in the estimation of language models [Lavrenko and Croft 2001].

The final similarity of a textual document $d$ to a query $q$ is estimated by the following expression:

$$
p(q \mid d)=\prod_{w \in q}(\lambda p(w \mid c)+(1-\lambda) p(w \mid d))
$$


where $p(w \mid c)$ and $p(w \mid d)$ stand for the likelihood of observing word $w$ in collection $c$ and a textual description $d$, respectively. $\lambda$ is a smoothing parameter and is chosen empirically. We followed the suggestion in [Zhai and Lafferty 2002] by setting $\lambda$ close to 1 . The setting of this parameter is further verified based on the monolingual information retrieval task using the data provided by ImageCLEF. The retrieval accuracy of the language model with $\lambda=0.9$ is around $50 \%$, which is close to the best performance of monolingual text retrieval reported by participants of ImageCLEF.

Since each textual document $\mathrm{d}$ in our data consists of multiple fields, such as title and description, the word probability for each document $p(w \mid d)$ can be further expanded as the summation of contributions from different fields, i.e.,

$$
p(w \mid d)=\frac{\sum_{i} \operatorname{freq}\left(w, f_{i}(d)\right)}{\sum_{i}\left|f_{i}(d)\right|}
$$

where notation $f_{i}(d)$ stands for the $i$-th field for document $d$ and $f r e q\left(w, f_{i}(d)\right)$ represents the frequency of the word $w$ in the $i$-th field of document $d$. $\left|f_{i}(d)\right|$ is the length of the $i$-th field for document $d$.

Both probability $p(w \mid d)$ and $p(w \mid c)$ can be estimated empirically, namely first counting the occurrence of word $w$ in field $f_{i}(d)$ and collection $c$, and dividing it by the length of $f_{i}(d)$ and $c$.

Furthermore, to account for the negative feedback from the user, we also constructed a negative language model. In particular, for a given negative word $w$ in the feedback query, the probability of not observing word $w$ in a document $d$, denoted by $p(\bar{w} \mid d)$, is computed as follows:

$$
p(\bar{w} \mid d)=\lambda^{\prime} p(\bar{w} \mid c)+\frac{1-\lambda^{\prime}}{(|d|+1)|V|}
$$

where $|V|$ is the vocabulary size. In the above, the probability mass $1 /(|d|+1)$ is assigned to account for the unseen words. In practice, we set $\lambda^{\prime}=\lambda=0.9$.

\subsection{Dataset}

The dataset used in our studies is Eurovision St Andrew collection (provided by the St Andrews University Library ${ }^{2}$ ) through CLEF (Cross Language Evaluation Forum $)^{3}$ [Clough et al. 2005]. This is a collection of around 30,000 photographs with significant historic value. Each image has a semi-structured caption that consists of information such as the title, the textual description of the image content, the date when the photograph was taken, and the location of the photograph, etc.

The following is an excerpt from [Clough et al. 2004] about the statistics of this dataset:

The dataset used consisted 28,133 historic photographs from the library at St Andrews University [Reid 1999]. All images are accompanied by a caption consisting of 8 distinct fields which can be used individually or collectively to facilitate image retrieval. The 28,133 captions consist

\footnotetext{
${ }^{2}$ http://www-library.st-andrews.ac.uk/

${ }^{3}$ http://ir.shef.ac.uk/imageclef2004/interactive.html

ACM Transactions on Information Systems, Vol. V, No. N, September 2006.
} 
of 44,085 terms and 1,348,474 word occurrences; the maximum caption length is 316 words, but on average 48 words in length. All captions are written in British English and contain colloquial expressions and historical terms. Approximately $81 \%$ of captions contain text in all fields, the rest generally without the description field. In most cases the image description is a grammatical sentence of around 15 words. The majority of images (82\%) are black and white, although colour images are also present.

In our experiment, we only used the description field for the retrieval purposes.

\subsection{User Interface}

We implemented two interfaces as shown in Figure 1. Figure 1(a) is a manual refinement interface. Through this interface, users can freely refine their queries (i.e., adding or removing query terms) at each iteration based on the retrieved results. Figure 1(b) is the term feedback interface. In this interface, two lists of terms are shown to the user. The first list of terms captures all the query terms that have been used so far. The users can de-select any terms from this list after seeing the retrieved results. The second list of terms is automatically generated by the system at each iteration based on the user's prior queries and the retrieved results ${ }^{4}$.

Users can choose terms from this list to further refine their queries. Having both de-selecting list and selecting list, the term feedback interface is comparable to the manual refinement interface where users can freely add or remove their query terms at each iteration. For both the manual refinement interface and the term feedback interface, a user is required to provide an initial query to start the search. At each iteration, both interfaces show the twenty top retrieved images. The only difference between two interfaces is query refinement as described above.

Figure 2 shows an example of using the two interfaces to find a target image. For both of these interfaces, the same retrieval model described in Section 3.1 is used to retrieve images based on the query terms. The search stops after five minutes (i.e., the stopping criteria for the interaction). Figure 2(a) shows the target image and its description. Figure 2(b) shows an interaction session for the manual refinement interface. The target image is found within seven iterations in this example. Figure 2(c) shows an interaction session for the term feedback interface. In this example, we can see cases where a relevant term is not selected by the user, for example, hills in iteration 1 and harbour in iteration 2 . We can also see cases

\footnotetext{
${ }^{4}$ Suppose our system retrieves 100 images based on a query. The top 20 images which are shown to the user belong to a Top Set and the remaining 80 images belong to a Bottom Set. The system currently generates terms based on the entropy of a term in the image descriptions in the Bottom Set. The ten terms with highest entropy are put in the list.This strategy focuses on the ability of a term to separate the set of images that are not yet shown to the user, but could be related to the search topic. The stronger the ability is, the more efficiently this term helps the system to narrow down the search space. We chose this strategy since it performed better than other strategies we experimented with. However, since the quality in term generation will affect the overall effectiveness of the term feedback interface, instead of further identifying better strategies for term generation, we did a series of simulation studies on the role of term generation rate in the utility of the term feedback interface (see Section 4.2).
} 


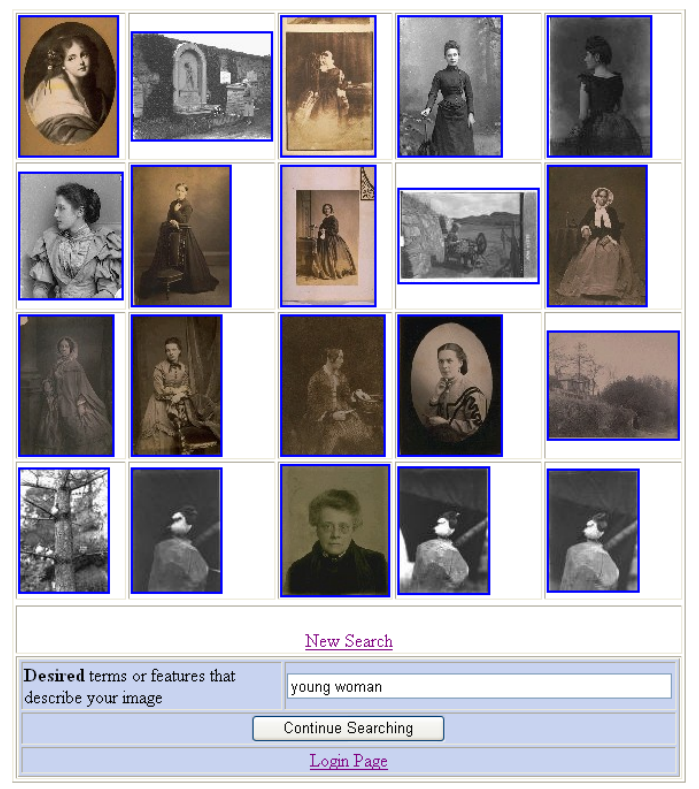

(a) Manual refinement interface

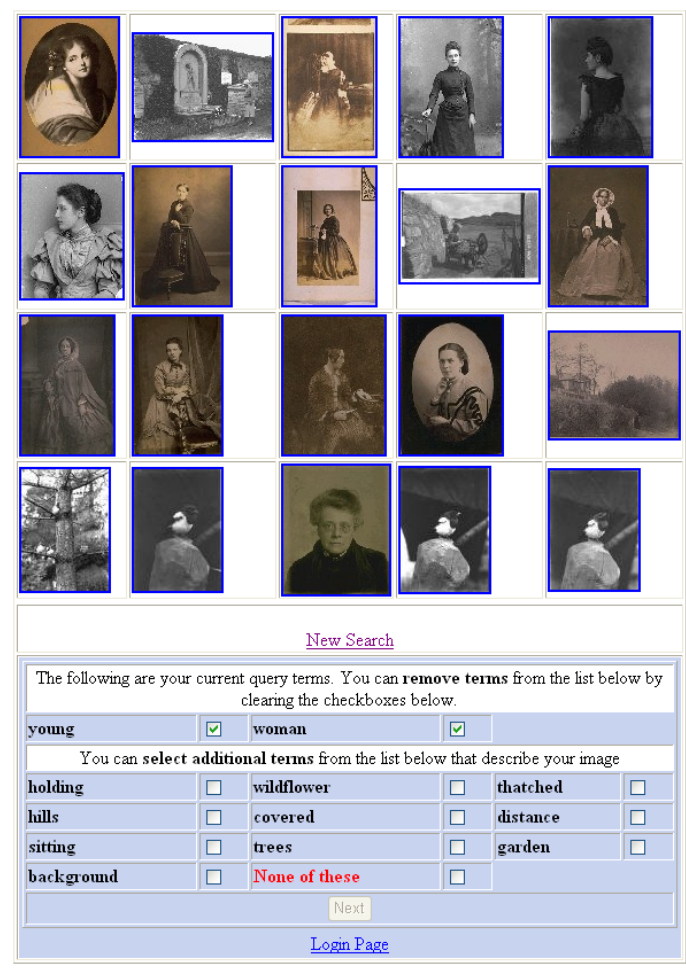

(b) Term feedback interface

Fig. 1. Two interfaces used in the study ACM Transactions on Information Systems, Vol. V, No. N, September 2006. 


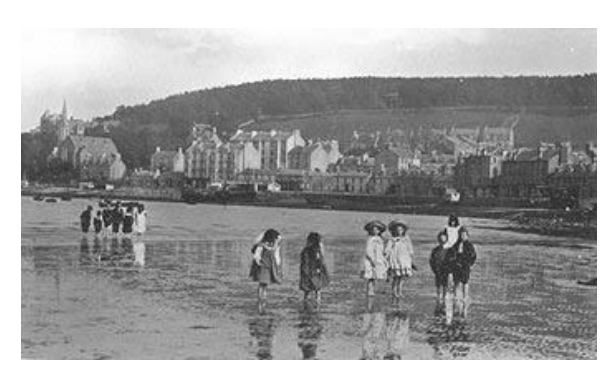

Description: Children paddling on the sea edge; boats in harbour, church, houses and shops round bay; hill with trees behind.

(a) The target figure and its description

Query 1: beach children mountain Result 1: (system showing 20 images) Query 2: beach children mountain Result 2: (system showing 20 images) Query 3: beach children mountain boats Result 3: (system showing 20 images) Query 4: beach children mountain water Result 4: (system showing 20 images) Query 5: beach children mountain barefoot Result 5: (system showing 20 images) Query 6: beach children mountain twins Result 6: (system showing 20 images) Query 7: beach children mountain port Result 7: (system showing 20 images) Target Found!

(b) Manual refinement interface
Query 1: beach children mountain

Result 1: (system showing 20 images and prompting term list: holidaymakers, town, hills, huts, rowing, oval, bathing, road, distance, benches)

User selects nothing

Query 2: children beach

Result 2: (system showing 20 images and prompting term list: shore, woods, steep, backed, harbour, chairs, woman, esplanade, gardens, deck)

User selects: shore

Query 3: children beach shore

Result 3: (system showing 20 images and prompting term list: loch, village, track, waterfront, foreground, whitewashed, cottages, pier, breaking, machines)

User selects: waterfront

Query 4: children beach shore waterfront

Result 4: (system showing 20 images and prompting term list: foreshore, changing, street, end, lines, open, pool, shoreline, car, rough)

User selects: open

User deselectes: waterfront, shore

Query 5: children beach open

Result 5: (system showing 20 images and prompting term list: man, train, swimmers, spectators, lawn, girls, seaside, formal, taking, crane)

Target not found! Task failed!

(c) Term feedback interface

Fig. 2. An example of an interaction session for each interface

that the user selects a term that is not in the description of the target image, such as shore in iteration 2 and waterfront in interaction 3 . In this example, the retrieval task fails after five iterations because 5 miniutes have elapsed.

\subsection{Study Methodology}

Eight subjects participated in the study. Each of them was asked to search for 16 images (see Appendix) provided by the interactive track at ImageCLEF. The subjects were first asked to complete a screening questionnaire to elicit demographic data and data concerning their search experience. Then, the subject was asked to use one interface to search eight images (one at a time). After using each interface, the subject was given a questionnaire to indicate how easy he/she felt about the search process, and how satisfied he/she was with a particular system. The sequence of which image and which interface to use was predefined based on the Latin Square design in order to guarantee balance between system order and image order. During each search, the system also automatically logged information such as the initial query from a user, the system retrieved results, terms prompted by the system, 


\begin{tabular}{|l|l|l|}
\hline & Manual refinement & Term feedback \\
\hline \hline Success Rate Overall & 0.48 & 0.27 \\
\hline Average Time per Target Image & $1: 41$ & $2: 41$ \\
\hline \hline Average No. of Iterations per Target Image & 4.3 & 4.1 \\
\hline \hline
\end{tabular}

Table I. Overall performance comparison of two interfaces

and the time spent on searching, etc. When an image was found or when the five minute time limit ran out, the search stopped. After searching all images using two different interfaces, each subject was asked to give an overall ranking of the two interfaces in terms of their overall satisfaction and both systems' effectiveness of locating the target images.

\subsection{Experimental Results}

Before conducting the user study, our expectation was that the term feedback interface should work better than the manual refinement interface. This expectation was partly based on our hypotheses and partly based on results from previous studies on term feedback in text retrieval.

However, our experimental results indicate otherwise. Table I shows the comparative results for the two interfaces. Since two out of the sixteen images do not have textual descriptions (which are the only information used to generate terms), the results reported here are based on the fourteen images. We consider a retrieval task successful if the target image is shown in the top 20 retrieved images. The successful retrieval rate is calculated by dividing the number of successful retrievals by the total number of retrieval attempts for that interface. As shown in Table I, the success rate for the term feedback interface is significantly lower than the success rate for the manual refinement interface (T-test, $p<0.05$ ). Among the successful retrievals, the average time spent on retrieving a target image in the term feedback interface is significantly higher than the time spent on the term manual refinement interface. This time difference is potentially due to two factors. The first factor relates to the system computation time required to generate terms in the term feedback interface. The second factor relates to the time taken by a user to provide feedback (i.e., either to manually refine terms or to select terms prompted by the system). In our current investigation, we have not made distinctions between those two contributing factors. Furthermore, as shown in Table I, among the successful retrievals, the average number of iterations taken to retrieve the target images by the term feedback interface is comparable to that taken by the manual refinement interface.

To further understand these results, we made an effect comparison between the two interfaces concerning the desired or undesired terms given by a user. Relevant terms are the query terms specified by the user that appear in the description of target images. Irrelevant terms are the query terms specified by the user that do not appear in the target descriptions ${ }^{5}$. Table II shows the average number and average percentage of relevant or irrelevant query terms (with respect to the total

${ }^{5}$ Note the definitions of relevant/irrelevant terms here are different from the conventional use. Here they indicate whether a term appears in the textual description of a target image.

ACM Transactions on Information Systems, Vol. V, No. N, September 2006. 


\begin{tabular}{|l|l|l|}
\hline & Manual refinement & Term feedback \\
\hline \hline Avg. percentage of relevant terms & $4.2 \%$ & $6.2 \%$ \\
\hline Avg. number of relevant terms & 0.53 & 0.79 \\
\hline Avg. percentage of irrelevant terms & $16.1 \%$ & $18.3 \%$ \\
\hline Avg. number of irrelevant terms & 1.44 & 2.18 \\
\hline \hline
\end{tabular}

Table II. Comparison of relevant terms between the manual refinement interface and the term feedback interface

number of terms in the description of the target image) that are specified among all iterations through each interface.

The results indicate that the average percentages of relevant terms are quite small for both interfaces. This reflects the difficulties involved for users to specify those relevant terms. As expected, the percentage of relevant terms is higher in the term feedback interface compared to the manual refinement interface. However, the percentage of irrelevant terms is also higher in the term feedback interface compared to the manual refinement interface. These observations imply that in terms of the ability to help users specify relevant terms, the term feedback interface works better than the manual refinement interface. However, when terms are provided by the system, there is also a tendency for users to select terms that are irrelevant to the target images. Furthermore, the percentage of irrelevant terms is higher than the percentage of relevant terms on average. The irrelevant terms seem to have a stronger impact on the final retrieval performance than the relevant terms. In other words, the negative effect appears to bypass the positive effect for the term feedback interface compared to the manual refinement interface. Therefore, we have not seen the advantage of term-feedback interface.

\subsection{Performance Analysis}

To better understand the positive and negative effects, we analyzed factors that contribute to these effects. We use two variables $P E$ (i.e., the positive effect) and $N E$ (i.e., the negative effect) to measure the utility of term feedback.

More specifically, for each target image $t$, a given initial query $q$, at each iteration $i$, there will be a $P E$ number and a $N E$ number. The $P E$ indicates the number of terms selected by the user that actually appear in the description of the target image (i.e., the relevant terms). The $P E$ can be further decomposed as the following three components:

$$
P E_{t, q, i}=N_{\text {list }} \times \text { GenRate }_{t, q, i} \times \text { SelRate }_{t, q, i}
$$

Here $N_{\text {list }}$ is the number of candidate terms shown in the term list. GenRateR is the generation rate for relevant terms, which indicates the percentage of terms in the term list that actually appear in the description of the target image. SelRateR is the relevant term selection rate, which measures the percentage of the relevant terms that are selected by a user.

Similarly, we can measure the negative effect $(N E)$ as follows:

$$
N E_{t, q, i}=N_{\text {list }} \times \text { GenRateIRR } R_{t, q, i} \times \text { SelRateIRR } R_{t, q, i}
$$

The $N E$ indicates the number of terms that are selected by the user but are not in the description of the target image (i.e., irrelevant terms). Here $N_{\text {list }}$ is the same 


\begin{tabular}{|l|c|}
\hline Average GenRateR & 0.06 \\
\hline \hline Average GenRateIRR & 0.94 \\
\hline Average SelRateR & 0.26 \\
\hline Average SelRateIRR & 0.08 \\
\hline \hline
\end{tabular}

Table III. Term generation rate and term selection rate for the term feedback interface

constant as described above. GenRateIRR is the irrelevant term generation rate, which measures the percentage of terms in the term list that do not appear in the description of the target image. SelRateIRR is the irrelevant term selection rate, which measures the percentage of irrelevant terms that are selected by the user.

It is worth mentioning that the term selection rate in one iteration can affect the term generation rate in the next iteration. To simplify the analysis, we did not take such in-between-iteration effects into consideration. Rather, we incorporated such effects in an average rate across all iterations. Table III shows the empirical results of these measurements from our user study. As indicated in Table III, although the percentage of selecting irrelevant term is relatively lower, because of the large number of irrelevant terms are generated, the overall selection of irrelevant terms is still much higher than the selection of relevant terms. More specifically, the product of the average GenRateR and the average SelRateR amounts to 0.016; while the product of the GenRateIRR and the average SelRateIRR amounts to 0.075. This indicates that the negative effect $N E$ is bigger than the positive effect $P E$, which is consistent with our experimental results shown in Table II.

Given ineffective term feedback in our system, the next question is whether this result is largely due to our current design of the system. In other words, is it because of the limitations of our system (e.g., algorithms to generate the list of terms), the effect of term feedback cannot be clearly demonstrated? If we made certain components better in our system, will the term feedback become more effective? With these questions in mind, we conducted further investigation on the potential of term feedback.

\section{POTENTIAL OF TERM FEEDBACK}

To go beyond our current system and understand the potential of term feedback for text-based image retrieval, we further investigated the three factors that contribute to the utility of term feedback interfaces: the term selection rate (i.e., SelRateR and SelRateIRR), the term generation rate (i.e., GenRateR and GenRateIRR), and the number of terms shown to the user $\left(N_{\text {list }}\right)$. In this section, we give a detailed account of the roles of these factors in term feedback interfaces.

\subsection{Term Selection}

One way to improve the utility of term feedback is by improving the term selection rate, namely, by increasing the chances of selecting the relevant terms from the term list (i.e., SelRateR) and decreasing the chances of selecting the irrelevant terms (i.e., SelRateIRR). Since term selection relates to user responses to prompted terms, one question is whether users respond to different terms differently. Some terms may have higher visual functions than others and thus are easier to be recognized by a user. For example, the term house could be easier to recognize than the term

ACM Transactions on Information Systems, Vol. V, No. N, September 2006. 
background. It seems relatively easier to tell whether there is a house in a target image than to tell what background is. Can we classify a set of terms that are more informative than the others in terms of the ease of recognition from a user's point of view with respect to an image? If we can identify the behavior of those informative terms, then we can design some mechanisms to give those terms higher priority to be included in the term list. Because supposedly users are more responsive to the informative terms, our hypothesis is that such classification can potentially increase SelRateR and decrease SelRateIRR.

To validate our hypothesis, we conducted a second user study to examine the potential classification of terms for the term selection purpose. 130 users participated in this study, which was conducted on the web. These users were mainly undergraduate students in the CSE department. There were 180 images used in the study. On average, each user searched for about 9 images. Each image was searched by six to seven different users.

In this study, each user interacted with the term feedback interface to find target images through the web. To search for one target image, a user had to first provide an initial text query in his/her own terms. The system would then retrieve the top 20 images and show them to the user. Along with the retrieved images, the system also prompted 10 terms for the user to choose from. The user would be able to refine his or her query by selecting any terms from this list. This process was repeated until the user found a target or gave up the search. During the search, the system kept a $\log$ of the entire interaction. Based on the logged data, we collected 2483 unique terms (which appeared in the term list) with a total of 38655 occurrences. Each occurrence of these terms was assigned to the following four groups:

Group A. The terms that are in the description of the target image and are also selected by the user. This group of terms is considered informative in the sense that if they occur in the description of the target image (i.e., if they are relevant to user's information needs), users can easily recognize these terms.

Group B. The terms that are in the description of the target image but are not selected by the user. This group of terms is considered uninformative in the sense that even those terms appear in the target description (i.e., relevant), users have a hard time recognizing them.

Group $C$. The terms that are not in the description of the target image but are selected by the user. This group of terms is considered overly-expressive in the sense that even these terms are not relevant (i.e., do not occur in the target description), they tend to be mistakenly selected by a user.

Group D. The terms that are not in the description of the target image and are not selected by the user. This group of terms is not particularly interesting for our purpose.

Given each instance of a term in the term list, based on the feedback from the user, the system knows exactly which group this term instance belongs to.

A summary of these categories is shown in Table IV. By separating terms into four groups, we were hoping to build a learning model to classify a term into three categories: informative, uninformative, and overly-expressive. Then, based on such classification, each term could be given a weight indicating the priority of being 


\begin{tabular}{|l|l|l|}
\hline & Selected & Not selected \\
\hline \hline In the description & Group A: informative & Group B: uninformative \\
\hline Not in the description & Group C: overly-expressive & Group D: Other \\
\hline \hline
\end{tabular}

Table IV. Four categories of term selection behavior

included in the term list for users to choose. Ideally, informative terms should be given higher weights and uninformative and overly-expressive terms should be given lower weights.

Figure 3 shows the results from the 200 most frequently occurring terms. Figure 3(a) shows the behavior of terms in Group A and Group B. We can see that almost every term appears more frequently in Group B than in Group A. In other words, almost every word appears more often as an uninformative term than as an informative one. This finding suggests that almost none of the terms can be categorized as easily recognizable. Furthermore, the frequency ratio between uninformative and informative is steady across all terms. This indicates that no term seems to be significantly more informative than the other. Figure 3(b) shows the similar behavior between terms in Group A and terms in Group C. It indicates that almost every term is equally confusing. For each term, on the one hand, when it occurs in the target description (i.e., relevant), it can be recognized. On the other hand, when it does not occur in the target description, it can also be mistakenly chosen. Two curves in Figure 3(b) also indicate that no term seems to be more overly expressive than the other.

As a conclusion, the empirical results from the second user study indicate that term classification based on single terms themselves will not be successful. For most terms that are relevant from a user's point of view, their chances of being used as descriptive words for target images is far less than 50\%. In Figure 4, we plot the probability for the 200 most frequent user selected terms to be used in the description of any target images. Only 17.1 out of 200 words actually have a $50 \%$ chance of occurring in the target descriptions. Therefore, including a selected term to expand the original textual query that is considered relevant by the user may not necessarily improve the accuracy of retrieved results.

In fact, terms by themselves do not show any strong indication as to how they will be perceived or recognized by a user. In the task of finding a target image, the content of the image and the image features play an important role on how a term is perceived. For example, the term tree is intuitively an informative term. But if the tree objects are not in the prominent position in the target image, a strong response to the term tree will not be expected. Furthermore, different users may have different responses to a set of terms. A term may be informative to one user but not to the other. For example, the term loch may not mean anything to a user who is not familiar with this term.

We also found that applying NLP techniques to purely recognize named entities may not lead to any improvement in term selection. Among all 14 images used in our first user study (Section 3), we found two images that have descriptions containing named entities. One named phrase is Celtic style and the other is related to Robert Burns. Our feeling is that the proper names may mean a lot to users who have some knowledge about the background of the images, e.g., the special architecture 


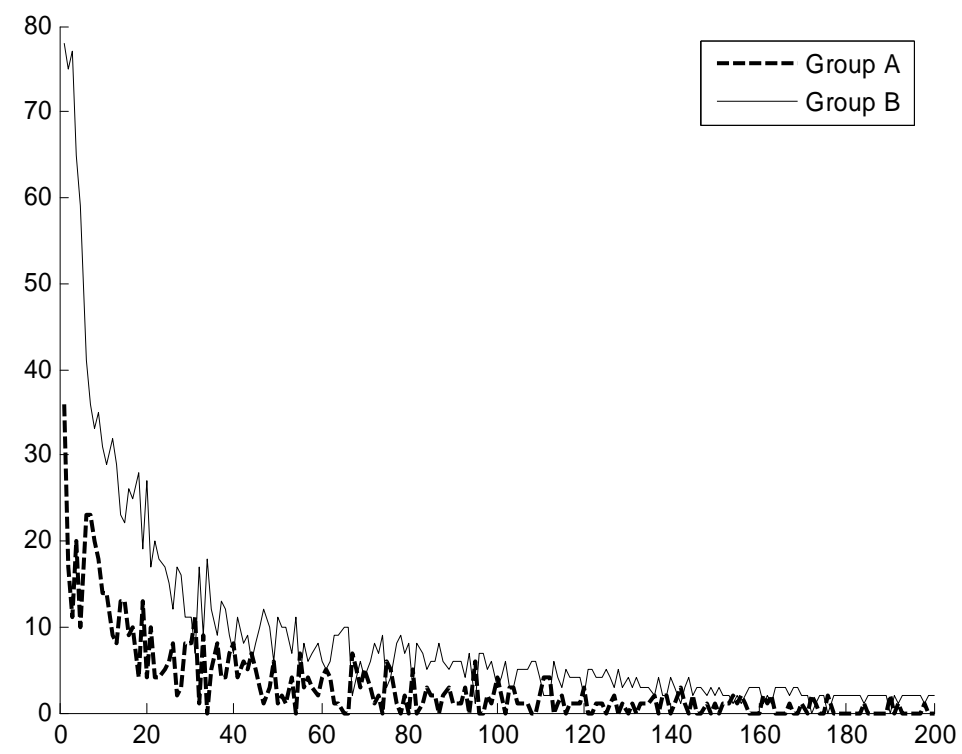

(a) Frequencies of terms being informative and uninformative

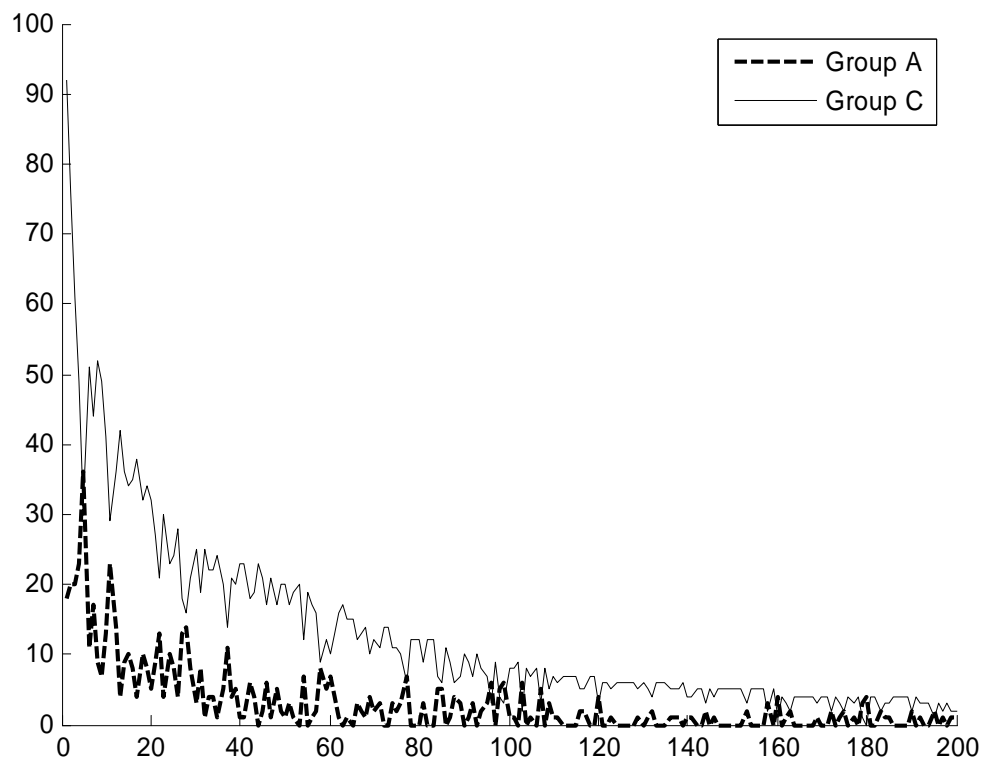

(b) Frequencies of terms being informative and overly-expressive

Fig. 3. Term selection comparison 


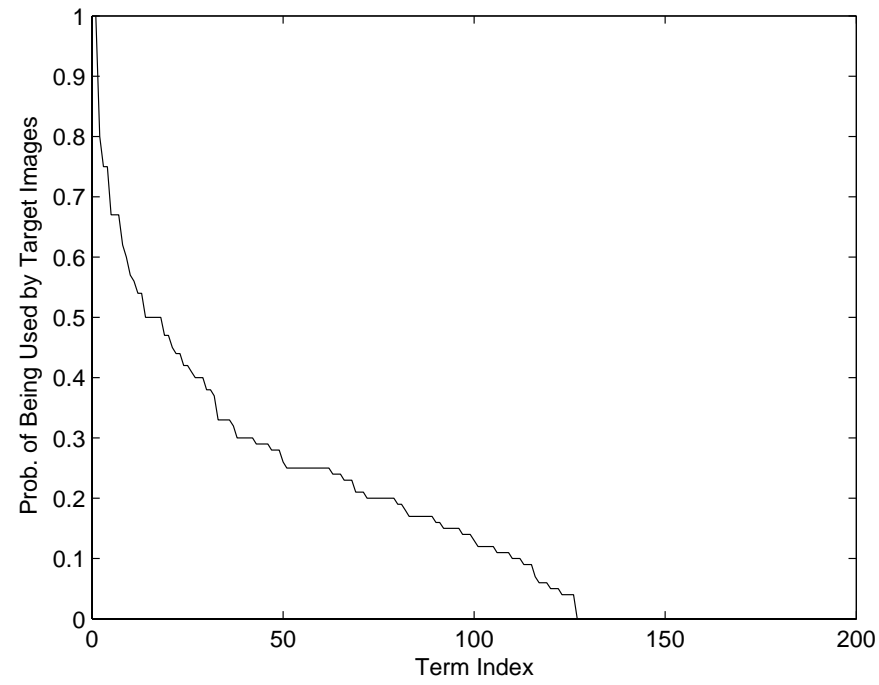

Fig. 4. Statistics on the selected terms that actually occur in the target description

style or the name of the person in the image. For people who do not have this type of background knowledge, providing proper names to the user may not make much difference. However, we believe that the proper names can be very helpful when the retrieved images and corresponding descriptions are shown to the user. In this case, the user can recognize the connections between those named entities with some image features and then use the same named entities to describe the similar image features in the target image.

Overall, our study indicates that term selection will not be improved only based on terms themselves. It requires more comprehensive study that takes into consideration terms, visual features of images, and user cognitive models of perception.

\subsection{Term Generation}

The second method focuses on improving the term generation rate, namely, increasing the relevant terms (i.e., GenRateR) and decreasing irrelevant terms in the term list (i.e., GenRateIRR). We have experimented with different term generation strategies [Zhang et al. 2005]. However, these different strategies (including the one described in Section 3) all resulted in roughly similar term generation rates (with about 0.06 as in Table III). The question now becomes whether it is this low relevant term generation rate that caused the unexpected low performance of term feedback as described in Section 3. In other words, if the relevant term generation rate is increased, will the utility of term feedback interfaces increase? What is the requirement on term generation in order for term feedback to reach reasonable performance?

To answer these questions, we examined the implications of different term generation rates on the overall retrieval performance based on the term feedback interface. In particular, we conducted a simulation study using the fourteen images in our 


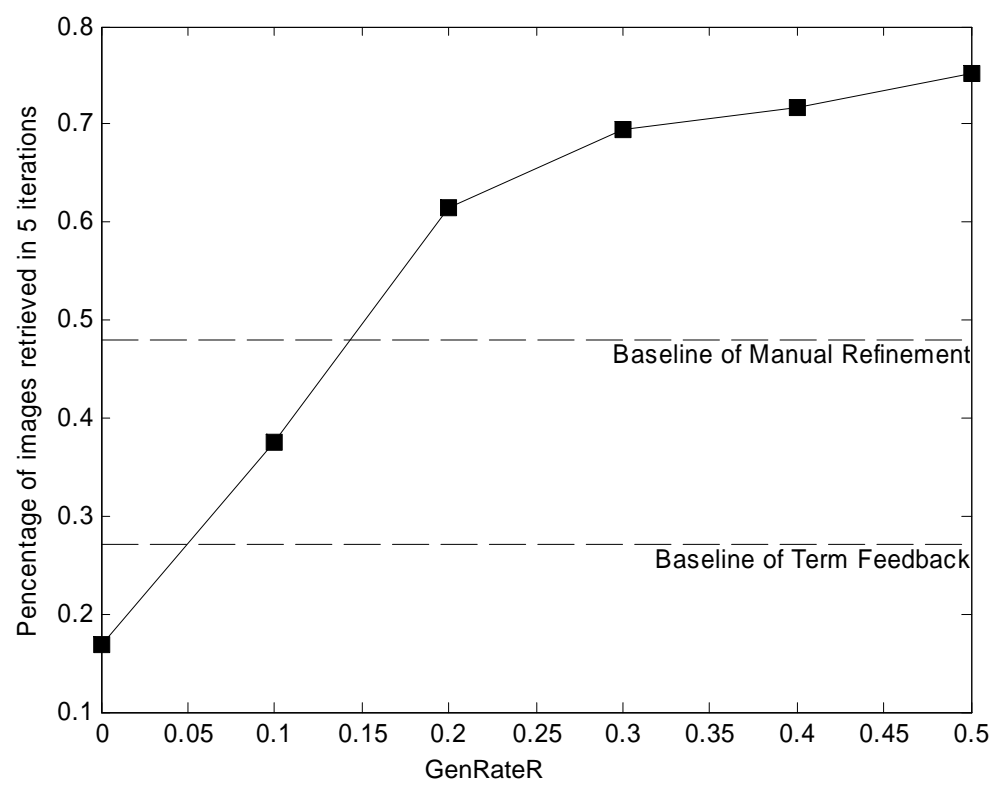

Fig. 5. Projected term feedback performance with respect to different relevant term generation rates

first user study (Section 3). For each image, we had 8 initial queries collected from the first user study. Therefore, the simulation was repeated 8 times, each time with a different initial query. These formed a total of $112\left(14^{*} 8\right)$ trials of image retrieval sessions.

For each trial, we simulated different term generation rates (i.e., GenRateR) without actually applying any term generation algorithm. At each iteration after the initial query, a list of 10 terms was generated. In that list, $(10 \times$ GenRateR $)$ relevant terms were randomly picked from the description of the target image, and the rest of the irrelevant terms were generated according to their term frequencies in the top retrieved image descriptions. The system also simulated the user term selection rate. Our study on term selection (Section 4.1) indicates that the term selection rate will not be easily improved without more sophisticated studies. Therefore, our system only applied the empirical rate of SelRateR and SelRateIRR (which are 0.26 and 0.08 , respectively, from Table III) to select the relevant/irrelevant terms from the term list to add to the query. The system then retrieved images based on the simulated queries. This retrieval process was repeated until a target image was retrieved or five iterations were reached.

Figure 5 shows the simulation results. Here, the $\mathrm{X}$-axis represents different levels of the term generation rate (GenRateR). The Y-axis represents the average percentage of target images that were successfully retrieved in five iterations among 112 trials. The curve in Figure 5 indicates that the final retrieval performance increases as the desired term generation rate increases. The two lines represent the performance of the term feedback interface and the manual refinement interfaces from the first user study (Table I). As we can see, the term feedback interface used 
in the first user study performs roughly at the same level as the generation rate 0.05 in the simulation. This is consistent with our previous results since the desired term generation rate for the term feedback interface used was 0.06 (see Table III). The manual refinement interface performs roughly at the same level as the generation rate of 0.15 . These results indicate that a considerable effort has to be made to improve the desired generation rate in order to make term feedback comparable to manual refinement.

\subsection{Number of Terms in the List}

The third method to improve the term feedback performance is to improve the number of terms in the prompted term list. The idea is that when the number of terms prompted to the user increases, users would have more opportunities to identify relevant terms.

To further investigate the role of the number of terms (i.e., $N_{\text {list }}$ ) prompted to the user at each iteration on term feedback performance, we conducted a further simulation study. In this study, we varied the number of terms that are prompted to the user from 5 to 20 . We did not go above 20 because a long list of terms will inevitably cause a huge cognitive burden on the user, which may not be practical. Results are shown in Figure 6. From this figure, before the relevant term generation rate reaches 0.2 , the performance increases as the number of terms prompted to the user increases. However, as the generation rate increases, the impact of $N_{\text {list }}$ diminishes. Once the relevant term generation rate reaches $0.2,10,15$, or 20 terms in the term list will not make a significant difference.

The results indicate that while effort has been put towards improving relevant term generation, increasing the number of terms in the prompted term list can have some advantages. Once the technologies for term generation are significantly advanced, the number of terms prompted to the user makes less effect. In practice, using only 5 terms in the list seems to be rather limited and should be avoided.

\subsection{Display Size}

The previous sections are concerned with the three different factors associated with the utility of term feedback. There are potentially other factors that can affect term feedback performance, but they have not been modeled in the utility measurement. One example is the display size of the retrieved results. Earlier studies have shown that people are able to scan a large number of images (thumbnails) and therefore methods of presenting images are important. To further investigate the role of display size in our particular searching task, we conducted another simulation study. In this simulation, the selection rate was set to 0.26 based on the first user study and Nlist was set to 10. Figure 7 shows the results. Here, the X-axis represents different term generation rates. Each curve corresponds to a display size ranging from 20 to 100. The simulation results have shown that, assuming that the user can easily glance through thumbnails to identify relevant images, the larger the display size, the more effective is the term feedback interface. Furthermore, the impact of the display size on the effectiveness of the interface diminishes as the display size increases incrementally. Because of this observation, it may not be necessary to present users with more than 100 thumbnails. Nevertheless, these results indicate that the display size is an important factor for interactive image search.

ACM Transactions on Information Systems, Vol. V, No. N, September 2006. 


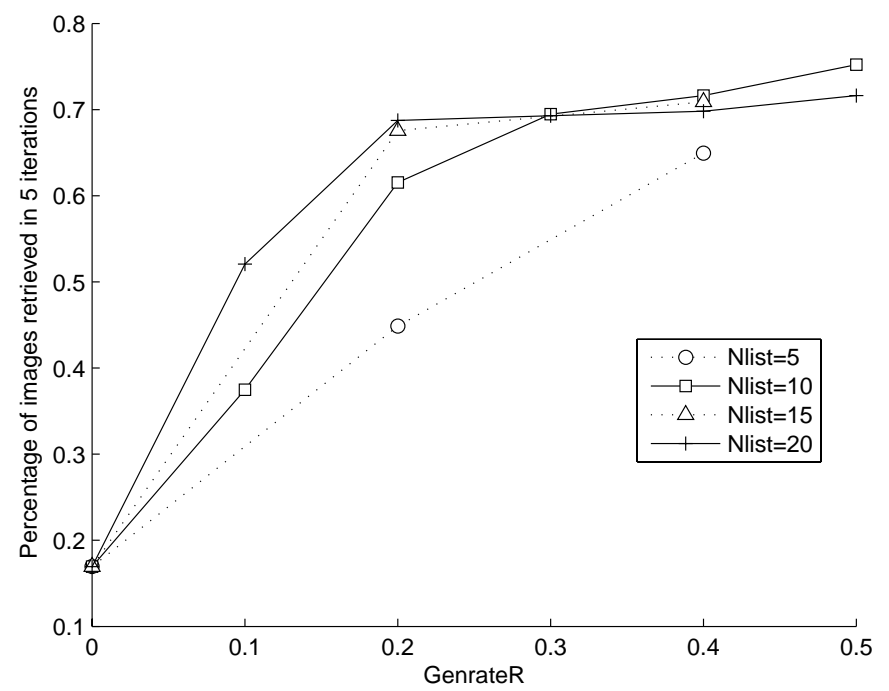

Fig. 6. Projected term feedback performance with respect to different Nlist

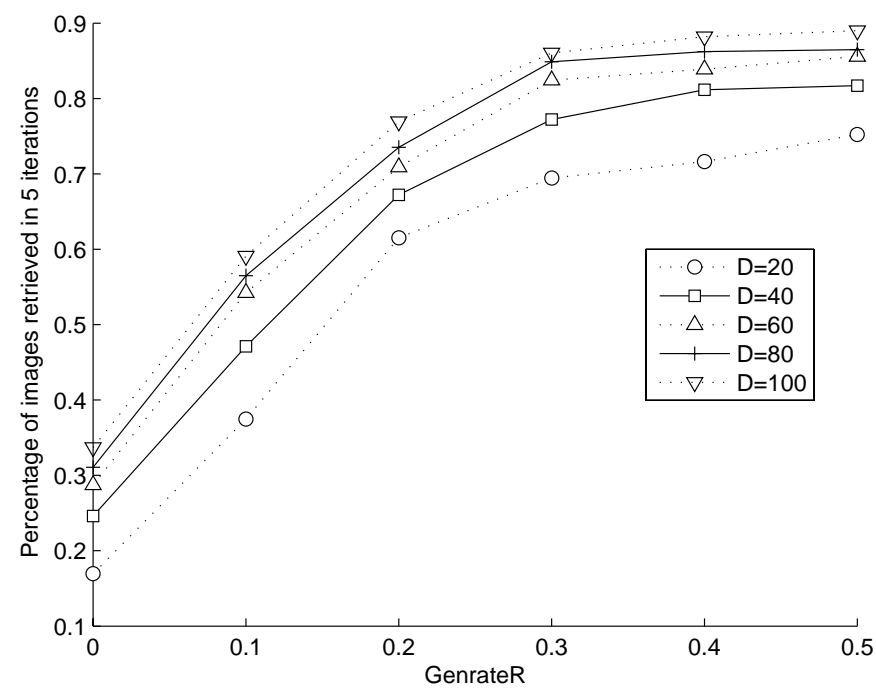

Fig. 7. Projected term feedback performance with respect to the display size 


\section{DISCUSSION AND CONCLUSION}

This paper presents our findings on the role of term feedback in interactive textbased image retrieval. In particular, we followed the ImageCLEF interactive track and focused on a special image searching scenario where users have a target in mind and try to find this target image from a collection of images. This scenario, in our view, is more challenging than the other general information seeking tasks since the search is only considered successful when the exact image is found. Anything that is similar to the exact image will not be counted as a successful retrieval.

In this unique setting, the results from our studies indicated that term feedback has not been as effective as we originally hypothesized. Although it has a positive effect by allowing users to identify more relevant terms, it also has a strong negative effect by providing more opportunities for users to select irrelevant terms. The negative affect appears stronger than the positive effect so that the overall benefit of term feedback in our text-based retrieval task is not significant.

To understand factors that contribute to these effects and their implications on the potential of term feedback, we conducted further investigation. Our studies indicate that the targeted search performance using term feedback is dependent on two main factors. (1) The term generation rate that applies to the underlying mechanism in generating relevant terms as candidate terms in the list. A considerable improvement in term generation has to be made in order to make the term feedback interface comparable to the current manual refinement interface. (2) The term selection rate that measures user responses to the prompted term list. It is shown that the term selection rate will be difficult to improve without more sophisticated studies that consider textual terms, image visual features, and user cognitive models of perceptions. In addition to these two main factors, our studies have shown that other issues such as the number of terms prompted by the system and the number of intermediate retrieved results presented to the user also play an important role in the effectiveness of interactive image search.

Note that the findings (e.g., Section 3) from our work are specific to our unique task, the backend data collection, and the implementation of our current system. As mentioned earlier, the task under investigation here is different from the general information seeking tasks. The St. Andrew collection is a difficult collection to search partly because of the use of colloquial language. Many terms in the descriptions may not be familiar to a user. Moreover, we currently only used descriptions for indexing. Past research on the St Andrews collection on interactive interfaces has shown that all caption text should be used for retrieval [Petrelli and Clough 2005]. Furthermore, in our current implementation, we did not show the descriptions along with each retrieved result. If we had descriptions provided for each image, we would expect even better performance for the manual refinement interface. The reason is that users can simply use the descriptions for the similar images as the textual query, which will usually result in higher performance. We have observed this behavior in a pilot study. Because of these various issues, it is not clear whether the findings obtained from our current investigation can be generalized to other information seeking tasks, data sets, or different implementations of the systems. Nevertheless, we believe that the methodologies developed in our current work can be applied to general investigations on interactive information

ACM Transactions on Information Systems, Vol. V, No. N, September 2006. 
seeking. We have learned that, in studying interactive technologies for information retrieval, it is desirable to apply both empirical user studies and simulation studies. While user studies can reveal user reactions and evaluate interactive systems, the simulation studies can provide a quick assessment on the potential utility before expensive implementation and user studies are taken place.

During interactive information search, different types of feedback can be solicited from a user. For example, previous work using relevance feedback has shown that allowing users to select one or more relevant images in the top $n$ retrieved results can improve cross-language image retrieval [Clough and Sanderson 2004]. Since the focus of our work is not on query expansion in general, but rather on a specific aspect that centers around how users are involved in refining queries, we have not yet experimented with any techniques related to relevance feedback. However, incorporating relevance feedback will certainly be an interesting aspect of our future work.

In addition, in our future work, we will investigate new approaches for interactive image retrieval that combines the strength of keyword-based approaches, contentbased image retrieval, and recent development in collaborative filtering [Jin et al. 2004]. Specifically, collaborative relevance judgments of images from all users, which were logged by CBIR systems, will be used as an auxiliary representation of images to address the semantic gap in the content-based approaches; multimodal user feedback, which allows users to provide not only relevance judgments but also preference feedback in both images and descriptive words, will be used to target the limitation of forming effective queries and query refinement.

\section{ACKNOWLEDGMENT}

This work was supported by grant IRGP-03-42111 from Michigan State University. The authors would like to thank Vineet Bansal for his work on interfaces and anonymous reviewers for their helpful comments and suggestions.

\section{APPENDIX}

The fourteen images that were used in Section 3 and Section 4.2 and Section 4.3 are shown in Figure 8, except 8(b) and 8(d), which have no descriptions.

\section{REFERENCES}

Anick, P. And Tipirneni, S. 1999. The paraphrase search assistant: Terminological feedback for iterative information seeking. In Proceedings of SIGIR'99. Berkeley, CA, 153-159.

Belkin, N. J., Cool, C., Koenemann, J., NG, K. B., And Park, S. 1996. Using relevance feedback and ranking in interactive searching. In Proceedings of TREC4.

Belkin, N. J. And Marchetti, P. G. 1990. Determining the functionality and features of an intelligent interface to an information retrieval system. In Proceedings of SIGIR'90. Brussels, Belgium, 151-177.

Berger, A. AND LAfferty, J. 1999. Information retrieval as statistical translation. In Proceedings of the 1999 ACM SIGIR Conference on Research and Development in Infor-mation Retrieval. $222-229$.

Bjarnestam, J. 1998. Description of an image retrieval system. In Proceedings of the Challenge of Image Retrieval Research Workshop.

Blei, D. And Jordan, M. 2003. Modeling annotated data. In Proceedings of 26th International Conference on Research and Development in Information Retrieval (SIGIR). 127-134. 


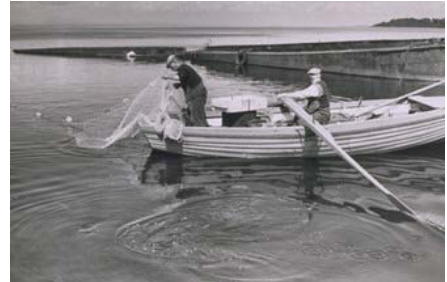

(a)

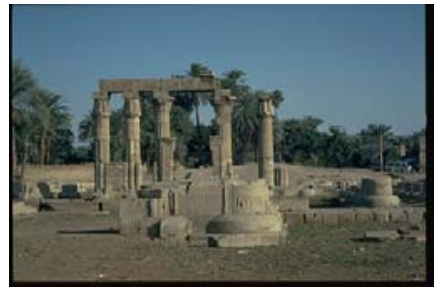

(d)

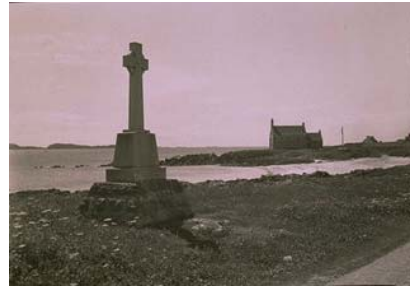

(g)

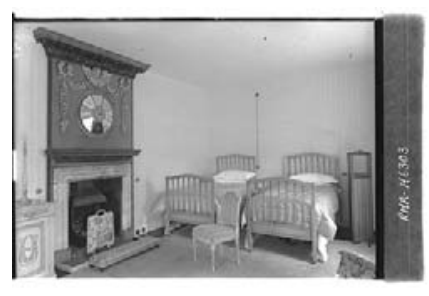

(j)

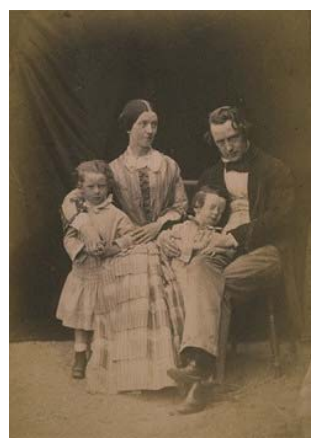

(m)

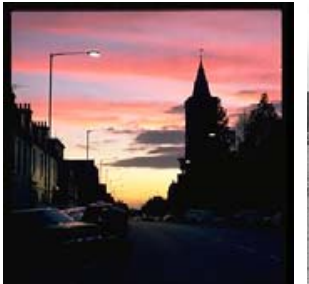

(b)

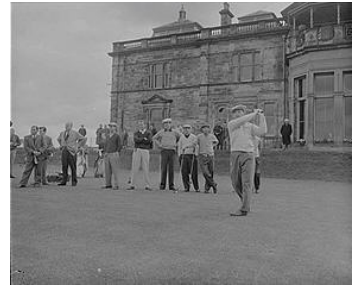

(e)

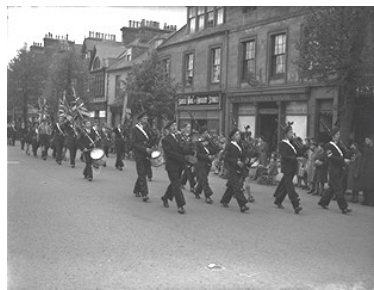

(h)

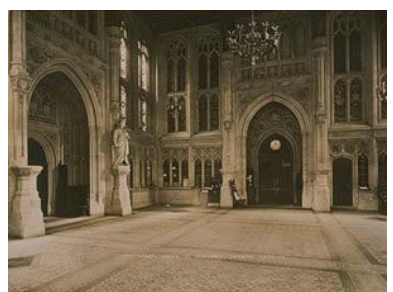

$(\mathrm{k})$

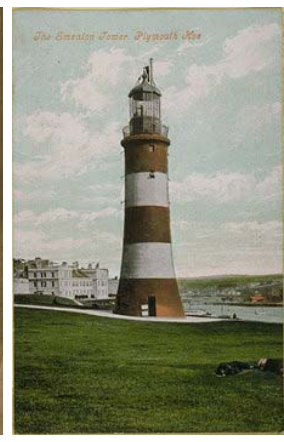

(n)

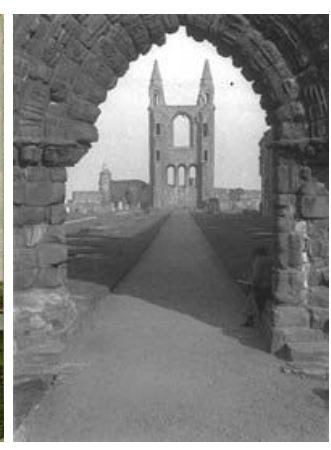

(o)

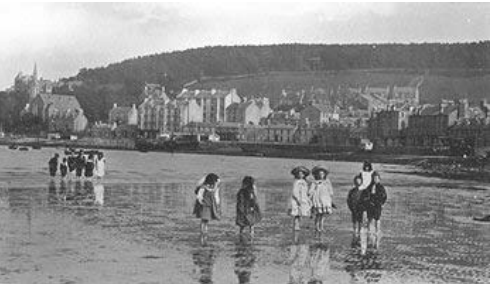

(c)

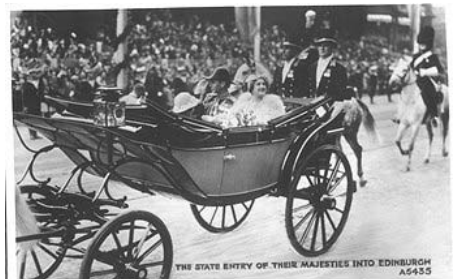

(f)

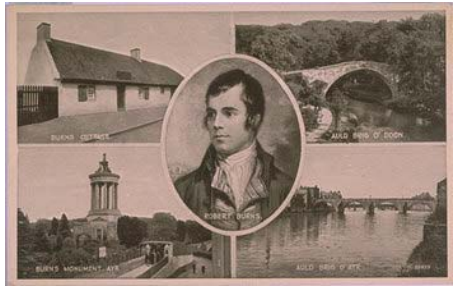

(i)

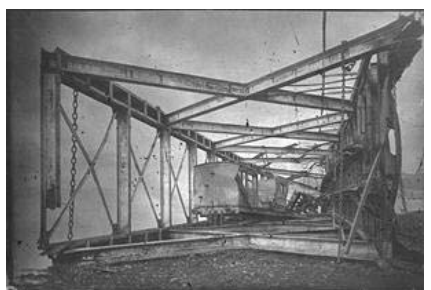

(1)

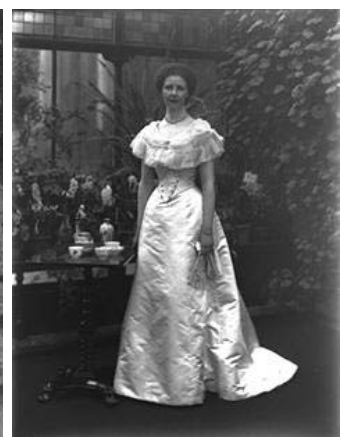

(p)

Fig. 8. Sixteen target images

ACM Transactions on Information Systems, Vol. V, No. N, September 2006. 
Chang, E. Y., Li, B., Wu, G., And Goh, K.-S. 2003. Statistical learning for effective visual information retrieval. IEEE International Conference on Image Processing (ICIP), 609-612.

Chang, S.-K. And Hsu, A. 1992. Image information systems: where do we go from here? IEEE Transactions on Knowledge and Data Engineering 17, 5, 431-442.

Chen, Y., Wang, J. Z., And KRVEtz, R. 2005. CLUE: cluster-based retrieval of images by unsupervised learning. IEEE Transactions on Image Processing 14, 8.

Choi, Y. And Rasmussen, E. 2003. Search for images: the analysis of user's queries for image retrieval in american history. Journal of the American Society for Information Science and Technology 54, 6 (April), 498-511.

Clough, P., Mueller, H., and Sanderson, M. 2005. The CleF 2004 Cross language Image Retrieval Track. Multilingual Information Access for Text, Speech and Images: Results of the Fifth CLEF Evaluation Campaign, Lecture Notes in in Computer Science LNCS 3491/2005, 597-613.

Clough, P. And Sanderson, M. 2004. The effects of relevance feedback in cross language image retrieval. Proceedings of the 26th European Conference on IR Research (ECIR'04), 238-252.

Clough, P., Sanderson, M., and Mueller, H. 2004. A proposal for the ClEF Cross-Language Image Retrieval Track 2004. Conference for Video and Image Retrieval (CVIR 2004).

Clough, P., Sanderson, M., And Reid, N. 2003. The eurovision st andrews photographic collection (esta). Image clef report, University of Sheffield, UK. February.

Enser, P. G. B. 1995. Pictorial information retrieval. Journal of Documentation 51, 2, 126-170.

Evgeniou, T., Pontil, M., Papageorgiou, C., and Poggio, T. 2003. Image representations and feature selection for multimedia database search. IEEE Transactions on Knowledge and Data Engineering 4, 911-920.

Faloutsos, C., Barber, R., Flicker, M., Hafner, J., Niblack, W., Petkovic, D., and Equitz, W. 1994. Efficient and effective querying by image content. Journal of Intelligent Information Systems 3, 231-262.

FEDER, J. 1996. Towards image content-based retrieval for the world-wide web. Avanced Imaging 11, 1, 26-29.

Forsyth, D. 1997. Finding pictures of objects in large collections of images. In Digital Image Access and Retrieval: 1996 Clinic on Library.

Frankel, C., Swain, M. J., And Athitsos, V. 1997. Webseer: An image search engine for the world wide web.

Harman, D. 1988. Towards interactive query expansion. In Proceeding of SIGIR'88. 321-331.

He, X., King, O., Ma, W.-Y., Li, M., And Zhang, H. J. 2003. Learning a semantic space from user's relevance feedback for image retrieval. IEEE Transaction on Curcuits and Systems for Video Technology 13, 1 (January), 39-48.

He, X., Ma, W.-Y., And Zhang, H.-J. 2004. Learning an image manifold for retrieval. In Proceedings of ACM MM 2004.

Hearst, M. 1994. Using categories to provide context for full-text retrieval results. In Proceedings of RIAO'94.

HenNiger, S. AND Belkin, N. 1996. Interface issues and interaction strategies for information retrieval systems. In Proceedings of the Human Factors in computing Systems Conference (CHI'96). ACM Press, New York, 401-402.

Hoi, C.-H. AND Lyu, M. R. 2004a. A novel log-based relevance feedback technique in contentbased image retrieval. In Proceedings of ACM MM 2004. ACM Press, New York, NY, USA.

Hoi, C.-H. AND LyU, M. R. 2004b. A novel log-based relevance feedback technique in contentbased image retrieval. In Proc. of the 12th ACM International Conference on Multimedia. $24-31$.

Jeon, J., Lavrenko, V., and Manmatha, R. 2003a. Automatic Image Annotation and Retrieval using Cross-Media Relevance Models. In Proceedings of the 26th annual international ACM SIGIR. 
Jeon, J., Lavrenko, V., and Manmatha, R. 2003b. Automatic image annotation and retrieval using cross-media relevance models. In Proceedings of the 26th annual international ACM SIGIR conference on Research and development in informaion retrieval. 119-126.

Jin, R., Chai, J., And Si, L. 2004. An automated weighting scheme for collaborative filtering. In Proceedings of The 27th Annual International ACM SIGIR Conference on Research and Development in Information Retrieval (SIGIR 2004). Sheffield, UK, 337-344.

Jorgensen, C. 1996. Indexing images: Testing an image description template. In ASIS 1996 Annual Conference Proceedings.

Keister, L. H. 1994. User types and queries: impact on image access systems. In Challenges in indexing electronic text and images, R. Fidel, P. J. Smith, T. B. Hahn, and E. M. Rasmussen, Eds. ASIS, 7-22.

Koenemann, J. And Belkin, N. J. 1996. A case for interaction: a study of interactive information retrieval behavior and effectiveness. In Proceedings of the SIGCHI Conference on Human Factors in Computing Systems: Common Ground. Vancouver, British Columbia, Canada, 205212 .

Lafferty, J. And Zhai, C. X. 2001. Document language models, query models, and risk minimization for information retrieval. In Proceedings of The 24th Annual International ACM SIGIR Conference on Research and Development in Information Retrieval. 111-119.

Lavrenko, V. And Croft, B. 2001. Relevance-based language models. In Proceedings of the 24th Annual International ACM SIGIR Conference on Research and Development in Information Retrieval. $120-127$.

Lavrenko, V., Manmatha, R., And Jeon, J. 2003. A model for learning the semantics of pictures. In Proceedings of Advance in Neutral Information Processing.

Li, J. AND WANG, J. Z. 2003. Automatic linguistic indexing of pictures by a statistical modeling approach. IEEE Trans. on Pattern Analysis and Machine Intelligence 25, 19, 1075-1088.

McDonald, S. And TAit, J. 2003. Search strategies in content-based image retrieval. In Proceedings of SIGIR 2003. Toronto, Canada, 80-87.

Miller, D. H., Leek, T., And Schwartz, R. 1999. A hidden markov model information retrieval system. In Proceedings of the 1999 ACM SIGIR Conference on Research and Development in Information Retrieval. 214-221.

Monay, F. and Gatica-Perez, D. 2003. On image auto-annotation with latent space models. In Proc. ACM International Conference on Multimedia. 275-278.

Ogle, V. E. And Stonebraker, M. 1995. Chabot: retrieval from a relational database of images. IEEE Computer 28, 9, 40-48.

Pentland, A., Picard, R. W., And Sclaroff, S. 1996. Photobook: Tools for content-based manipulation of image databases. International Journal of Computer Vision 18, 3, 233-254.

Petrelli, D. And Clough, P. 2005. Concept hierarchy across languages in text-based image retrieval: a user evaluation. Working notes of the CLEF Workshop.

Ponte, J. 1998. A language modeling approach to information retrieval. Ph.D. thesis, Department of Computer Science, Univ. of Massachusetts at Amherst.

Ravela, S. And Manmatha, R. 1998. Retrieving images by appearance. In Proceedings of IEEE International Conference on Computer Vision (ICCV98). 608-613.

REID, N. 1999. The photographic collections in St Andrews University Library. Scottish Archives 5, 83-90.

Rui, Y., HuAng, T. S., AND Chang, S.-F. 1999. Image retrieval: Current techniques, promising directions, and open issues. J. Visual Comm. and Image Representation 10, 1, 39-42.

Rui, Y., Huang, T. s., Ortega, M., and Mehrotra, S. 1998. Relevance feedback: A power tool in interactive content-based image retrieval. IEEE Trans. on Circuits and Systems for Video Technology, Special Issue on Segmentation, Description, and Retrieval of Video Content 8, 5 (September), 644-655.

SAntini, S. 2001. Exploring image databases context-based retrieval. Academic Press, San Diego, USA.

Shatford Layne, S. 1994. Some issues in the indexing of images. In Journal of American Society for Information Science. Vol. 45. 583-588.

ACM Transactions on Information Systems, Vol. V, No. N, September 2006. 
Shen, H. T., OoI, B. C., And Tan, K. L. 2000. Giving meanings to www images. In Proceedings of ACM MM. 39-48.

Shneiderman, B. And Kang, H. 2000. Direct annotation: A drag-and-drop strategy for labeling photos. In Proceeding of International Conference on Information Visualization (IV2000). London, England, 88-95.

Smeulders, A. M. W., Worring, M., Santini, S., Gupta, A., And Jain, R. 2000. Content-based image retrieval at the end of the early years. IEEE Trans. on Pattern Analysis and Machine Intelligence 22, 12 (December), 1349-1380.

SRIHARI, R. K., ZhANG, Z., AND RAO, A. 2000. Intelligent indexing and semantic retrieval of multimodal documents. Information Retrieval 2, 245-275.

Tamura, H. And Yokoya, N. 1984. Image database systems: A survey. Pattern Recognition 17, 1, $29-43$.

Vendrig, J., Worring, M., And Smeulders, A. 2001. Filter image browsing: Interactive image retrieval by using database overviews. Multimedial Tools and Applications 15, 83-103.

Wactlar, H. D., Kanade, T., Smith, M. A., And Stevens, S. M. 1996. Intelligent access to digital video: Informedia project. Computer 29, 5, 46-52.

Wang, J. Z., Li, J., And Wiederhold, G. 2001. Simplicity: Semantics-sensitive integrated matching for picture libraries. IEEE Transactions on Pattern Analysis and Machine Intelligence 23, 9, 947-963.

Zhai, C. And LAfFerty, J. 2002. Two-stage language models for information retrieval. In Proceedings of the 25th Annual International ACM SIGIR Conference on Research and Development in Information Retrieval (SIGIR'02). 49-56.

Zhang, C., Chai, J., And Jin, R. 2005. User term feedback in interactive image retrieval. In Proceedings of the 28th annual international ACM SIGIR conference on Research and development in information retrieval. 51-58. 